\title{
Indications and Outcome in Patients Undergoing Left Atrial Appendage Closure-The Austrian LAAC Registry
}

\author{
David Zweiker ${ }^{1,2, *(1)}$, Raphael Sieghartsleitner ${ }^{1}$, Lukas Fiedler ${ }^{3}{ }^{(D}$, Gabor G. Toth ${ }^{1}$, Olev Luha ${ }^{1}$, \\ Guenter Stix ${ }^{4}$, Harald Gabriel ${ }^{4}$, Paul Vock ${ }^{5}$, Brigitte Lileg ${ }^{5}$, Andreas Strouhal ${ }^{6}$, \\ Geort Delle-Karth ${ }^{6}$, Michael Pfeffer ${ }^{3}$, Josef Aichinger ${ }^{7}{ }^{D}$, Wolfgang Tkalec ${ }^{7}$, \\ Clemens Steinwender ${ }^{8}$, Kurt Sihorsch ${ }^{8}$, Ronald K. Binder ${ }^{9}$, Martin Rammer ${ }^{9}$, \\ Fabian Barbieri ${ }^{10}{ }^{\circledR}$, Silvana Mueller ${ }^{10}$, Nicolas Verheyen ${ }^{1}$, Klemens Ablasser ${ }^{1}$, Andreas Zirlik ${ }^{1}$ \\ and Daniel Scherr 1,11 \\ 1 Department of Cardiology, Medical University of Graz, 8036 Graz, Austria; rsieghar@hotmail.com (R.S.); \\ gabor.g.toth@medunigraz.at (G.G.T.); olev.luha@klinikum-graz.at (O.L.); \\ nicolas.verheyen@medunigraz.at (N.V.); klemens.ablasser@medunigraz.at (K.A.); \\ andreas.zirlik@medunigraz.at (A.Z.); daniel.scherr@medunigraz.at (D.S.) \\ 2 Third Department for Cardiology and Intensive Care, Klinik Ottakring, 1160 Vienna, Austria \\ 3 Department of Internal Medicine, Cardiology and Nephrology, Hospital Wiener Neustadt, \\ 2700 Wiener Neustadt, Austria; flukas@me.com (L.F.); michaelpfeffer@gmx.at (M.P.) \\ 4 Department of Internal Medicine II, Medical University of Vienna, 1090 Vienna, Austria; \\ guenter.stix@meduniwien.ac.at (G.S.); harald.gabriel@meduniwien.ac.at (H.G.) \\ 5 Department of Internal Medicine III, University Hospital St. Pölten, 3100 St. Pölten, Austria; \\ paul.vock@aon.at (P.V.); office@lileg-kardiologie.at (B.L.) \\ 6 Department of Cardiology, Hospital Nord-Klinik Floridsdorf, 1210 Vienna, Austria; \\ Andreas.Strouhal@gesundheitsverbund.at (A.S.); georg.delle-karth@gesundheitsverbund.at (G.D.-K.) \\ 7 Department of Internal Medicine II, Elisabethinen Hospital, 4020 Linz, Austria; \\ Josef.Aichinger@ordensklinikum.at (J.A.); wolfgang.tkalec@ordensklinikum.at (W.T.) \\ 8 Department of Cardiology, Kepler University Hospital, 4020 Linz, Austria; \\ hcsteinwender@hotmail.com (C.S.); kurt.sihorsch@kepleruniklinikum.at (K.S.) \\ 9 Department of Internal Medicine II, Klinikum Wels-Grieskirchen, 4600 Wels, Austria; \\ Ronald.Binder@klinikum-wegr.at (R.K.B.); martin.rammer@klinikum-wegr.at (M.R.) \\ 10 Department of Internal Medicine III, Medical University of Innsbruck, 6020 Innsbruck, Austria; \\ fabian.barbieri@i-med.ac.at (F.B.); silvana.mueller@tirol-kliniken.at (S.M.) \\ 11 Department of Cardiology, Cardiovascular Research Institute Maastricht (CARIM), Maastricht University \\ Medical Centre, 6229 ER Maastricht, The Netherlands \\ * Correspondence: david.zweiker@medunigraz.at; Tel.: +43-664-865-0460
}

Received: 21 September 2020; Accepted: 10 October 2020; Published: 13 October 2020

Abstract: Background: Complete real-world data on the indications and outcomes of left atrial appendage closure (LAAC) outside of clinical trials are rare. In this study, we stratified patients undergoing LAAC by indication groups. Methods: This analysis of the national multicentre Austrian LAAC Registry comprised all patients that underwent LAAC up until 2018 at the currently active centres in Austria. The baseline characteristics, procedural details and outcomes between the following indication groups were compared: bleeding as an indication for LAAC ("bleeding" group) vs. thromboembolism despite oral anticoagulation (OAC; "thromboembolism" group) vs. an intolerance to OAC for reasons other than the above ("other" group). Results: The analysis included 186 patients, with $59.7 \%$ in the "bleeding" group, $8.1 \%$ in the "thromboembolism" group and $32.2 \%$ in the "other" group. The $\mathrm{CHADS}_{2}$ score was the highest in the "thromboembolism" group and the HAS-BLED score was the highest in the "bleeding" group. The procedural outcomes were similar between groups (implantation success, $97.3 \%$ ), with major complications occurring in $7.0 \%$ of 
patients. One-year survival free from stroke, bleeding or LAAC-associated hospitalisation was $83.9 \%$, $90.0 \%$ and $81.4 \%$ in the "bleeding", "thromboembolism" and "other" groups, respectively $(p=0.891)$. Conclusions: In routine clinical practice, LAAC was used in a heterogeneous patient population with atrial fibrillation $(\mathrm{AF})$ and contraindication, inefficacy or intolerance to $\mathrm{OAC}$. The long-term outcome was favourable in all groups.

Keywords: atrial fibrillation; left atrial appendage; registry; stroke; bleeding

\section{Introduction}

Left atrial appendage closure (LAAC) has evolved as an alternative to oral anticoagulation (OAC) for stroke prevention in atrial fibrillation (AF) patients. This procedure is currently recommended in patients with AF, elevated stroke risk, and contraindications to long-term anticoagulation treatment (class IIb, level of evidence B) [1,2]. The European guidelines especially suggest that patients with a history of bleeding without reversible cause should be considered for LAAC [1]. However, in everyday clinical practice, patients with AF might have alternative indications as well, such as "intolerance" to OAC. This "intolerance" of OAC may range from a history of severe bleeding associated with $\mathrm{OAC}$ intake to personal patient preference. As a result, the treated patient population may be very heterogeneous regarding its comorbidities and risk profile. For example, the Evaluating Real-Life Clinical Outcomes in Atrial Fibrillation Patients Receiving the Watchman Left Atrial Appendage Closure Technology (EWOLUTION) registry reports a history of major bleeding only in the minority of patients [3]. As of today, there is only limited evidence regarding different indications for LAAC and their effects on procedural and long-term outcomes in daily clinical practice. The goal of the present study was therefore to evaluate the patient selection, outcomes and efficacy based on different indications of all patients that were included in the Austrian LAAC Registry until the end of 2017.

\section{Materials and Methods}

This study was an analysis of the prospective Austrian LAAC Registry (NCT03409159). This registry was initiated by the Austrian Society of Cardiology and comprises all LAAC procedures performed until the end of 2017 in all currently active centres in Austria. The ethics committee of the Medical University of Graz approved the study (29-355 ex 16/17). Information on the clinical follow up was completed using data from the Austrian government's population registry.

\subsection{Recruitment and Indications}

The recruitment of patients for LAAC was left to the discretion of the treating physician and the participating centres. All centres were advised to select patients suitable for LAAC based on current guidelines [1]. For further analysis, patients were stratified by the primary indication leading to the decision to perform LAAC. All patients with a history of major bleeding were categorised into the bleeding group. The second group (thromboembolism) comprised all patients with a history of cerebral or peripheral thromboembolism, with or without OAC. All residual patients were aggregated in the other group. Patients with both bleeding and thromboembolism events were stratified according to the index event on which basis the decision to perform LAAC was made.

\subsection{Procedure}

The selection of the device was left to the operators' or institutes' discretion. All patients that were enrolled in the registry received either Watchman ${ }^{\mathrm{TM}}$ (Boston Scientific, Marlborough, MA, USA), Amplatzer Cardiac Plug ${ }^{\mathrm{TM}}$ (Abbott Laboratories, North Chicago, IL, USA) or Amplatzer Amulet ${ }^{\mathrm{TM}}$ (Abbott Laboratories) devices. Procedures were performed according to the vendors' standard operating protocol. 


\subsection{Antithrombotic Treatment}

Pre-, peri- and post-procedural antithrombotic regimens were chosen by the treating physicians after considering the manufacturers' guidelines, the patients' comorbidities and their individual risk profile.

\subsection{Follow-Up}

Patients were followed according to the respective protocols of the participating centres, including transoesophageal echocardiography (TOE) being performed 3-6 months after LAAC and further visits every 6 to 12 months to the implanting centre or peripheral institutions. The follow-up data were analysed until 31 December 2017.

\subsection{Data Collection}

The registry parameters were based on the European Heart Rhythm Association (EHRA)/European Association of Percutaneous Cardiovascular Interventions (EAPCI) consensus statement on LAAC [4]. The data collection was performed either by an external reviewer or by a local representative. All data were collected and analysed anonymously. In addition to the regular follow-ups, all available databases of hospital associations were searched for any readmissions at other hospitals. Mortality data were assessed via a search through the Austrian government's population registry (POPREG, Statistics Austria) [5]. The date of the last follow-up was defined as either the last clinical visit or the last day of available survival data according to the population registry.

\subsection{Endpoints}

Complications were defined as follows: procedural major complication-any procedural complication requiring an invasive intervention, procedural minor complication-any other procedural complication, cardiac tamponade-pericardial effusion requiring pericardiocentesis or surgery, access site complication-any access site complication requiring intervention, ischemic stroke-clinically relevant ischemic stroke according to current guidelines, shock-hypotension requiring catecholamines, cardiopulmonary resuscitation — cardiac arrest requiring cardiopulmonary resuscitation, acute kidney injury-an increase of serum creatinine by $\geq 1.5$ times from baseline within $<7$ days, bleeding — any bleeding requiring rehospitalisation, stroke or thromboembolism—any ischemic stroke or peripheral thromboembolism requiring hospitalisation, hospitalisation due to LAAC - any rehospitalisation that was either a direct consequence of the LAAC procedure or was caused by malfunction of the LAAC device.

We defined residual flow to the left atrial appendage as any colour Doppler flow $>1 \mathrm{~mm}$ in diameter as detected using TOE. The residual flow was stratified into minor flow ( $>1$ and $\leq 5 \mathrm{~mm}$ ) and major flow (>5 mm).

\subsection{Statistical Analysis}

We used IBM SPSS 20 (IBM, Armonk, NY, USA) for data analysis. Values were expressed as count (proportion in percent), mean \pm standard deviation or median (interquartile range), as appropriate. For bivariate analysis, we used ANOVA in normally distributed values (based on the Kolmogorov-Smirnov $\mathrm{Z}$ test); otherwise, the Kruskal-Wallis test was used. If a significant interaction between groups was observed, we performed post-hoc testing using the Kruskal-Wallis test and LSD. For categorical values, we used Fisher's exact test. The $p$-values were adjusted according to the Bonferroni correction, if appropriate. Unless stated otherwise, distinct $p$-values in the text refer to between-group differences.

To predict the number of bleeding and stroke events based on individual $\mathrm{CHA}_{2} \mathrm{DS}_{2}-\mathrm{VASc}$ and HAS-BLED scores, we used the data of Olesen et al. [6,7], as outlined by LaHaye et al. [8]. Where applicable, we used the adjusted score instead of the reported score. After the calculation of the 
individual risk, we adjusted it according to the length of observation. All individual risks were added together to predict the number of bleeding and thromboembolism events per patient-year for the whole population, which was then compared to the observed stroke and thromboembolism event rate.

\section{Results}

Between November 2010 to December 2017, 186 consecutive patients undergoing LAAC at nine centres in Austria were included in this analysis (Figure 1). A median of 17 procedures was performed at each centre, with a range from 8 to 43 procedures. This corresponded to a median LAAC implantation rate of 7.6 per year and centre (range, 1.1 to 12.3). Two patients of a currently inactive centre had to be excluded because the procedural and follow-up data could not be provided by the implanting centre. The median age was 75 (interquartile range, $70-79$ ) years and $37.6 \%$ were female. The baseline characteristics are shown in Table 1.

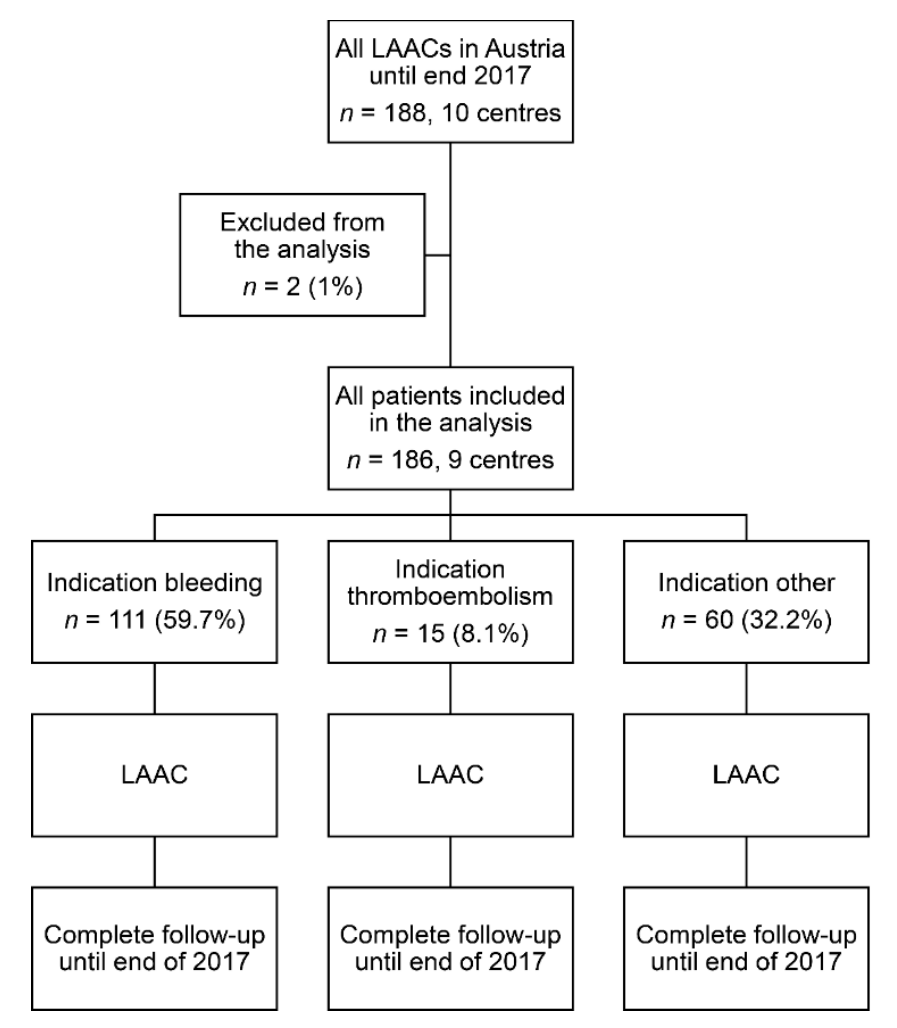

Figure 1. Study flowchart. LAAC: left atrial appendage closure.

Table 1. Baseline characteristics of the treated patients, grouped by their indication for LAAC.

\begin{tabular}{|c|c|c|c|c|c|}
\hline \multirow{2}{*}{ Parameter } & \multicolumn{3}{|c|}{ Indication for LAAC } & \multicolumn{2}{|c|}{$p$-Value } \\
\hline & Bleeding & Thromboembolism & Other & Overall & Post-Hoc \\
\hline Number of patients & 111 & 15 & 60 & $\mathrm{~N} / \mathrm{A}$ & \\
\hline Female & $38.7 \%$ & $20.0 \%$ & $40.0 \%$ & 0.355 & \\
\hline Body mass index, $\mathrm{kg} \mathrm{m}^{-2}$ & $26(24-30)$ & $25(22-30)$ & $27(24-31)$ & 0.409 & \\
\hline $\mathrm{CHA}_{2} \mathrm{DS}_{2}$-VASc score & $4.6 \pm 1.4$ & $4.8 \pm 1.2$ & $4.2 \pm 1.5$ & 0.150 & \\
\hline $\mathrm{CHADS}_{2}$ score & $2.8 \pm 1.2$ & $3.8 \pm 0.7$ & $2.5 \pm 1.2$ & $<0.001$ & $* *$, 拉 \\
\hline Arterial hypertension & $86.5 \%$ & $86.7 \%$ & $90.0 \%$ & 0.780 & \\
\hline Diabetes mellitus & $25.2 \%$ & $40.0 \%$ & $28.3 \%$ & 0.462 & \\
\hline Transitory ischemic attack, stroke or thromboembolism & $47.7 \%$ & $100.0 \%$ & $28.3 \%$ & $<0.001$ & $* *$, t㧊 \\
\hline Vascular disease & $42.3 \%$ & $20.0 \%$ & $38.3 \%$ & 0.270 & \\
\hline Uncontrolled hypertension & $9.0 \%$ & $0 \%$ & $8.3 \%$ & 0.762 & \\
\hline
\end{tabular}


Table 1. Cont.

\begin{tabular}{|c|c|c|c|c|c|}
\hline \multirow{2}{*}{ Parameter } & \multicolumn{3}{|c|}{ Indication for LAAC } & \multicolumn{2}{|c|}{$p$-Value } \\
\hline & Bleeding & Thromboembolism & Other & Overall & Post-Hoc \\
\hline Abnormal renal function & $15.3 \%$ & $6.7 \%$ & $13.3 \%$ & 0.803 & \\
\hline Abnormal hepatic function & $1.8 \%$ & $0 \%$ & $6.8 \%$ & 0.240 & \\
\hline History of bleeding & $100.0 \%$ & $26.7 \%$ & $45.0 \%$ & $<0.001$ & $* *,++$ \\
\hline Labile International Normalised Ratio values & $2.7 \%$ & $6.7 \%$ & $1.7 \%$ & 0.453 & \\
\hline Alcohol abuse & $2.7 \%$ & $0 \%$ & $3.2 \%$ & 0.655 & \\
\hline Cerebral artery disease & $15.0 \%$ & $13.3 \%$ & $10.9 \%$ & 0.837 & \\
\hline Periphery artery disease & $7.2 \%$ & $13.3 \%$ & $5.0 \%$ & 0.389 & \\
\hline History of percutaneous intervention & $22.5 \%$ & $0 \%$ & $31.7 \%$ & 0.021 & $\ddagger$ \\
\hline History of coronary artery bypass grafting & $13.5 \%$ & $6.7 \%$ & $5.0 \%$ & 0.200 & \\
\hline Chronic obstructive pulmonary disease & $18.2 \%$ & $6.7 \%$ & $7.3 \%$ & 0.133 & \\
\hline Dialysis & $1.0 \%$ & $0 \%$ & $0 \%$ & 1.000 & \\
\hline
\end{tabular}

Paroxysmal atrial fibrillation (AF) was defined as a sinus rhythm detected on the ECG shortly before the procedure. The following symbols represent significant differences in the post-hoc testing (after a Bonferroni adjustment): bleeding vs. embolism: ${ }^{*} p<0.05,{ }^{* *} p<0.01$; bleeding vs. other: ${ }^{\dagger} p<0.05,{ }^{+\dagger} p<0.01$; embolism vs. other: $\ddagger$ $p<0.05$, 㧊 $p<0.01 ; \S$ no significant interaction in the post-hoc testing found.

\subsection{Indications}

A history of bleeding was present in $59.7 \%(n=111)$ of patients (bleeding group), with most of the patients having suffered from intracranial bleeding (31.7\%, Figure 2). The rate of intracerebral haemorrhage $(23.1 \%)$ in this group exceeded other causes of intracranial bleeding (subdural hematoma: $4.8 \%$, subarachnoid bleeding: 3.8\%, epidural haemorrhage: $0.5 \%$, including patients with multiple intracranial bleedings). Gastrointestinal bleeding had occurred in $19.9 \%$, followed by epistaxis $(0.5 \%)$ and other $(7.0 \%)$. The minority of patients with a history of bleeding $(42.3 \%)$ had suffered a bleeding event without being on OAC; others had received either direct oral anticoagulants (DOACs) or vitamin K antagonists (VKAs, 28.85\% each).

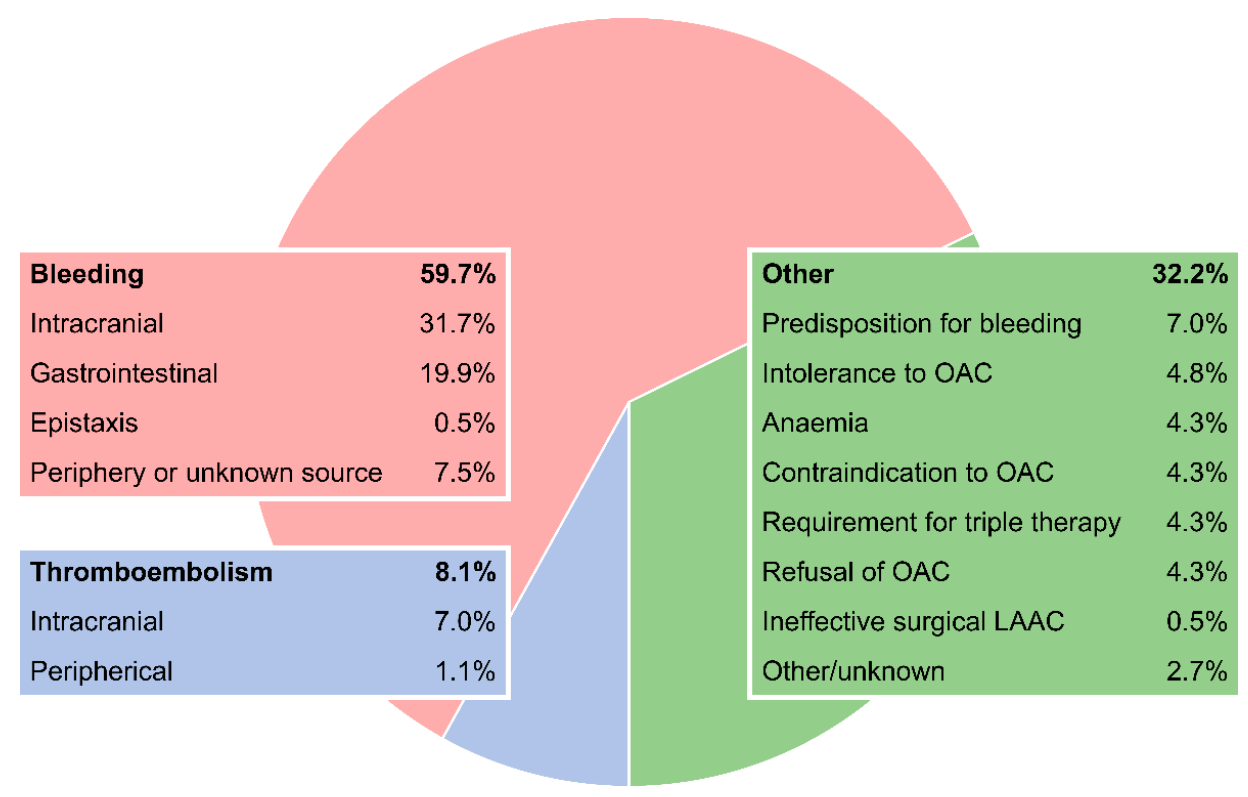

Figure 2. Indications of LAAC in the Austrian LAAC Registry. LAAC: left atrial appendage closure; OAC: oral anticoagulation.

A total of $8.1 \%$ of patients received LAAC because of a history of thromboembolism despite OAC treatment, with $7.0 \%$ having a history of stroke and $1.1 \%$ having a history of peripheral embolism (thromboembolism group). 
The third indication group (the other group) was very heterogeneous and comprised $32.2 \%$ of patients. Seven percent of patients had a predisposition for bleeding, such as gastrointestinal malformation (2.2\%), Morbus Osler (1.6\%) or cerebral malformation (1.1\%). Other indications were intolerance to an OAC due to side effects without major bleeding (4.8\%), anaemia without obvious cause $(4.3 \%)$ and contraindication to an OAC ( $4.3 \%$; for example, both liver and renal insufficiency). Another $4.3 \%$ of patients had LAAC because they refused a lifelong OAC and $4.3 \%$ received LAAC prior to a planned PCI to avoid triple antithrombotic therapy. One patient received LAAC to complete a previously performed insufficient surgical left appendage exclusion.

\subsection{Basic Risk Profile}

The $\mathrm{CHA}_{2} \mathrm{DS}_{2}$-VASc score of the overall cohort was $4.5 \pm 1.4$, the $\mathrm{CHADS}_{2}$ score was $2.8 \pm 1.2$ and the HAS-BLED score was $3.3 \pm 0.9$ (Table 1). Differences in comorbidities led to significantly different risk profiles between the pre-specified groups.

As expected, the HAS-BLED score was significantly higher in the bleeding group compared to the thromboembolism $(p=0.001)$ and other groups $(p<0.001)$ due to a more prevalent history of bleeding (100.0\% vs. thromboembolism $26.7 \%$ vs. other $45.0 \%, p<0.001)$.

Patients with a history of thromboembolism had a significantly higher thromboembolic risk (according to $\mathrm{CHADS}_{2}$ score) than the other group patients $(p<0.001)$ due to a significantly higher prevalence of stroke $(93.3 \%$ vs. $18.3 \%, p<0.001)$.

Coronary artery disease was significantly less prevalent in thromboembolism patients $(6.7 \%)$ compared to the other two groups (bleeding patients: $45.0 \%, p=0.013$; other patients: $50.0 \%, p=0.008$ ).

Patients in the other group had a significantly lower prevalence of transitory ischemic attack, stroke or thromboembolism (28.3\%) than the patients of other groups (bleeding group patients $47.7 \%$, thromboembolism group patients $86.7 \%$ ). Consequently, the $\mathrm{CHADS}_{2}$ score and HAS-BLED score were lowest in those patients.

\subsection{Antithrombotic Treatment before LAAC}

Of the whole patient population, $42.0 \%$ had received oral anticoagulation (DOAC, 29.6\%; VKA, $12.4 \%$ ) before the LAAC procedure (Table S1). The others had received low molecular weight heparin (LMWH, 23.7\%), aspirin (23.1\%), $\mathrm{P}_{2} \mathrm{Y}_{12}$ inhibitors (mostly clopidogrel, 14.0\%) or dual antiplatelet therapy $(8.1 \%)$. DOAC therapy prior to LAAC was significantly more prevalent in the thromboembolism group $(80.0 \%$ ) compared to the remaining groups (bleeding group $23.4 \%$, other group $28.3 \%, p<0.01$ ).

\subsection{Procedure}

The LAAC procedure was combined with other procedures in $14.3 \%$ of cases. The most frequent simultaneous procedure was the closure of a patent foramen ovale $(10.7 \%)$, which was performed numerically more often in the thromboembolism group $(26.7 \%)$ than in the bleeding group $(7.1 \%)$ and the other group $\left(12.7 \%, p_{\text {overall }}=0.055\right.$, Table S2). Other procedures were transcatheter mitral-valve repair $(1.8 \%)$ or coronary angiography and/or percutaneous coronary intervention $(1.8 \%)$. Cases were almost equally shared between the Amplatzer Cardiac Plug ${ }^{\mathrm{TM}}$ or Amplatzer Amulet ${ }^{\mathrm{TM}}$ device $(52.2 \%)$ and the Watchman ${ }^{\mathrm{TM}}$ device (46.8\%). More details about the procedure and procedural outcome can be found in Table S2.

\subsection{Procedural Outcome}

The median diameter of the implanted devices was $25 \mathrm{~mm}$ (IQR 24-27). The LAAC device was implanted successfully during the first procedure in $97.3 \%$ of patients. The reasons for implantation failure were technical difficulties $(n=3,1.6 \%)$, intraprocedurally detected LAA thrombus $(n=1,0.5 \%)$ and dislocation due to LAA anatomy $(n=1,0.5 \%)$. The procedure duration (without other concomitant procedures) ranged from $20 \mathrm{~min}$ to $3.5 \mathrm{~h}$ (median, $70 \mathrm{~min}$ ). Medians of 15 minutes (interquartile range, 
11-23) of fluoroscopy and $100 \mathrm{~mL}$ (66-148) of contrast were needed, without significant differences between the indication groups or between devices.

Major complications occurred in $7.0 \%$ of patients, with no significant differences between any of the groups. The most common complications were cardiac tamponade $(3.2 \%)$, access site complication $(2.2 \%)$, ischemic stroke $(1.1 \%)$ and shock requiring catecholamines $(1.1 \%)$. The procedural and in-hospital mortality was $0 \%$.

When stratified by centre, periprocedural complications ranged from $0 \%$ to $25 \%$. There was no correlation between the periprocedural complication rate and the implantation rate per year and centre $(\rho=-0.201, p=0.604)$.

\subsection{Antithrombotic Treatment after LAAC}

After the LAAC procedure, a majority of patients (52.7\%) were prescribed dual antiplatelet therapy (mostly clopidogrel plus aspirin) for 1-6 months after LAAC (Table S3). Other patients received an OAC with a DOAC (14.0\%), OAC with a VKA (5.9\%), single antiplatelet therapy with aspirin $(7.5 \%)$, single antiplatelet therapy with clopidogrel (5.4\%) or no antithrombotic therapy at all $(12.4 \%)$. Oral anticoagulants were prescribed significantly more often in the thromboembolism group than in the bleeding group $(53.3 \%$ vs. $17.1 \%, p=0.004)$. For OAC, phenprocoumon $(17.1 \%)$, apixaban $(46.4 \%)$, dabigatran $(35.7 \%)$ or rivaroxaban $(17.9 \%)$ were used.

After a median of 3 months (IQR 2-6), 43.5\% of patients switched to a single antiplatelet drug regimen as a long-term therapy. In $39.2 \%$ of patients, no further antithrombotic therapy was prescribed. Only in the thromboembolism group, four patients (26.7\% of all thromboembolism patients) switched to an OAC with a DOAC $(20.0 \%)$ or a VKA $(6.7 \%)$. The long-term antithrombotic therapy could not be determined in $15.1 \%$ of all patients.

\subsection{Follow-Up}

Data from follow-up TOEs, performed $96 \pm 73$ days after the LAAC procedure, were available for $59.7 \%$ of patients. A correct position of the LAAC device was documented in $98.9 \%$ of cases. However, in one patient, the LAAC device could not be detected in the LAA and was found in a pulmonary vein. In another patient, the position was deemed incorrect due to a large gap of $6 \mathrm{~mm}$ between the device and the LAA wall. A minor residual flow was found in $1.8 \%$ of patients and a thrombus attached to the device in $1.9 \%$.

Long-term follow-up data were available for a mean of $477 \pm 464$ days, with $85.5 \%$ of patients reaching a follow-up after 90 days. In total, a combined $16.5 \%$ of the followed patients reached an endpoint of death, stroke, bleeding or LAAC-associated hospitalisation within the first year after the procedure, with no significant differences between indication groups (Table 2, Figure 3). During follow-ups, the all-cause mortality was $11.3 \%$, while bleeding occurred in $7.0 \%$, thromboembolism in $2.7 \%$, ischaemic stroke in $1.6 \%$ and transient ischaemic attack in $0.5 \%$. Patients that suffered a stroke or peripheral thromboembolism after LAAC were on single antiplatelet therapy $(55.6 \%)$, no antithrombotic therapy $(33.3 \%)$ or dual antiplatelet therapy $(11.1 \%)$ during the event. Bleeding occurred in patients receiving single antiplatelet therapy $(46.2 \%)$, dual antiplatelet therapy $(30.8 \%)$ or no antithrombotic therapy $(23.1 \%)$.

Three patients $(1.6 \%)$ had to be readmitted to a hospital due to LAAC-device-related complications: One patient suffered from Dressler's syndrome and one patient was admitted to the hospital for the administration of intravenous unfractionated heparin because of a large thrombus detected on the device using transthoracic echocardiography. In the third patient, a previously correctly implanted LAA occluder dislocated spontaneously and was found free-floating in a pulmonary vein. It had to be surgically removed. There was no significant difference between the indication groups in terms of the echocardiography results or long-term follow-up events. 
Table 2. Follow up after LAAC.

\begin{tabular}{ccccc}
\hline Parameter & \multicolumn{3}{c}{ Indication for LAAC } & $p$-Value \\
\cline { 2 - 5 } & Bleeding & Thromboembolism & Other & Overall \\
\hline $\begin{array}{c}\text { Follow-up duration (days) } \\
\text { Combined endpoint (1 year death, } \\
\text { stroke, bleeding or LAAC-associated }\end{array}$ & $474 \pm 449$ & $268 \pm 203$ & $535 \pm 525$ & 0.178 \\
hospitalisation) & $16.1 \%$ & $10.0 \%$ & $18.6 \%$ & 0.891 \\
$\quad$ Death & & & $16.7 \%$ & 0.253 \\
Bleeding & $9.0 \%$ & $6.7 \%$ & $8.3 \%$ & 0.668 \\
Stroke, transient ischaemic attack or & $7.2 \%$ & $0.0 \%$ & $6.7 \%$ & 0.762 \\
thromboembolism & $4.5 \%$ & $0.0 \%$ & $1.7 \%$ & 1.000 \\
Ischemic stroke & $1.8 \%$ & $0.0 \%$ & $0.0 \%$ & 1.000 \\
Transient ischaemic attack & $0.9 \%$ & $0.0 \%$ & $5.0 \%$ & 0.572 \\
Thromboembolism & $1.8 \%$ & $0.0 \%$ & $1.7 \%$ & 0.256 \\
Hospitalisation due to LAAC & $0.9 \%$ & $6.7 \%$ & $30.0 \%$ & 0.879 \\
Any hospitalisation & $28.8 \%$ & $33.3 \%$ & &
\end{tabular}

LAAC: left atrial appendage closure.

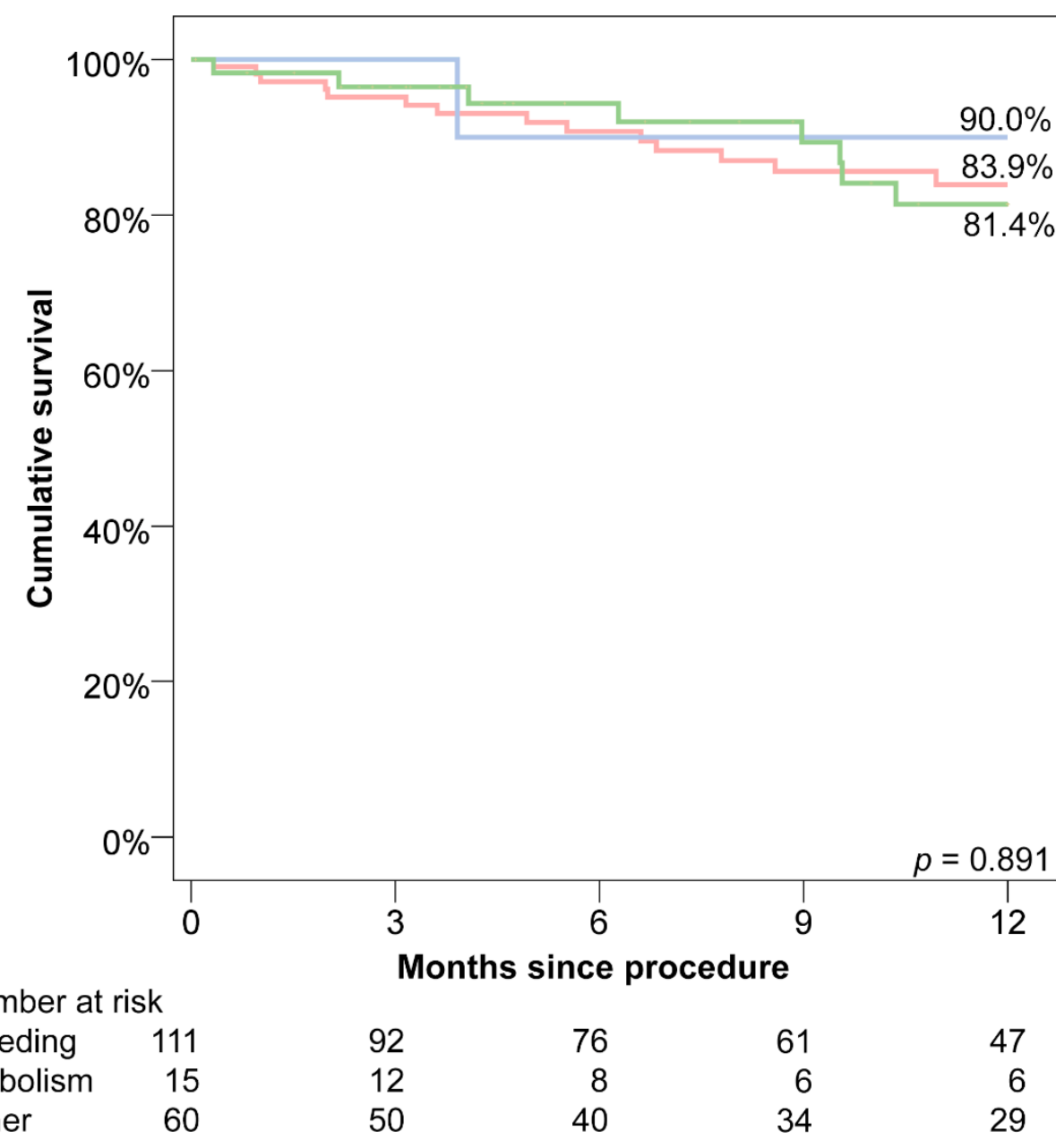

Figure 3. Kaplan-Maier curves for the combined endpoint of death, stroke, bleeding or LAACrelated rehospitalisation.

\subsection{Predicted vs. Observed Events}

Based on the $\mathrm{CHA}_{2} \mathrm{DS}_{2}$-VASc score of our patient cohort, we predicted a yearly rate of stroke or peripheral thromboembolism of $8.6 \%$. The predicted rate was the highest in the thromboembolism 
group (9.6\%). Within the observation period of our study, we found an annual event rate of 3.7\%, reflecting a significant relative reduction of $57 \%$ compared to the predicted rate ( $p=0.035$, Table 3$)$.

Table 3. Mean predicted annual stroke and bleeding events (as by $\mathrm{CHA}_{2} \mathrm{DS}_{2}$-VASc and HAS-BLED scores) compared to reported events in the Austrian LAAC Registry.

\begin{tabular}{ccccc}
\hline \multirow{2}{*}{ Parameter } & All Patients & \multicolumn{3}{c}{ Indication for LAAC } \\
\cline { 3 - 5 } & & Bleeding & Thromboembolism & Other \\
\hline Annual embolic events & & & & \\
Predicted & $8.6 \%$ & $8.9 \%$ & $9.6 \%$ & $7.8 \%$ \\
Observed & $3.7 \%$ & $3.5 \%$ & $0.0 \%$ & $4.5 \%$ \\
Relative reduction & $-57.0 \%$ & $-61.0 \%$ & $-100.0 \%$ & $-42.0 \%$ \\
$p$-Value & $0.035 *$ & 0.083 & 1.000 & 0.529 \\
Annual bleeding events & & & & \\
Predicted & $7.7 \%$ & $8.3 \%$ & $6.6 \%$ & $6.7 \%$ \\
Observed & $5.3 \%$ & $5.5 \%$ & $0.0 \%$ & $5.7 \%$ \\
Relative reduction & $-30.2 \%$ & $-33.2 \%$ & $-100.0 \%$ & $-15.6 \%$ \\
$p$-Value & 0.454 & 0.483 & 1.000 & 1.000 \\
\hline
\end{tabular}

LAAC: left atrial appendage closure. ${ }^{*} p<0.05$

According to the HAS-BLED score, our patient population had a predicted bleeding rate of $7.7 \%$ per year, with the highest predicted rate in the bleeding group patients $(8.3 \%)$. However, we observed a yearly bleeding rate of $5.3 \%$, leading to a relative risk reduction of $30 \%$, which did not prove to be statistically significant $(p=0.454)$. The observed annual rate of both thromboembolic events and bleeding events was highest in the other group (embolic events, $4.5 \%$; bleeding events, $5.7 \%$ ) and lowest in the thromboembolism group (no events during follow-up).

\section{Discussion}

This analysis of the Austrian LAAC registry revealed three major findings: First, in clinical practice LAAC was performed in a heterogeneous patient population with a high risk of bleeding or thromboembolism and varying individual risk profiles. Second, LAAC was associated with a considerable risk of procedural complications, which were all managed successfully. Third, long-term thromboembolic and bleeding rates after LAAC were lower than expected compared to published historic controls.

\subsection{Indications for $L A A C$}

The current study highlights that all patients receiving LAAC in Austria had an elevated risk of thromboembolism and bleeding, as measured by validated scores. The mean $\mathrm{CHA}_{2} \mathrm{DS}_{2}-\mathrm{VAsc}$ score was 4.5 and the mean HAS-BLED score was 3.3 across all groups. Therefore, the medical or non-medical strategies used to prevent thromboembolic events were justified in these patients.

As LAAC is currently indicated as a second-line therapy, a specific index event or condition usually leads to the decision to perform LAAC. This especially applies in Austria because only a certain number of LAAC procedures each year are reimbursed. As a result, these "indications" for LAAC are well documented and LAAC can only be done for the highest risk population. In this study, the patients' indications, which led to LAAC, were very heterogeneous. While bleeding (especially during OAC treatment) was the main reason in almost $60 \%$ of patients, in some patients, the predisposition for bleeding, refusal of an OAC or even the requirement for triple antithrombotic therapy for a limited duration were considered "contraindications" to OAC. Interestingly, $8.1 \%$ of patients received LAAC because they had experienced thromboembolic events, despite being on an adequate OAC.

There were significant differences in the baseline characteristics between indication groups, which could partly be explained by the indications themselves. However, there remained some 
other differences, such as a low incidence of coronary artery disease in the thromboembolism group, which endorses the importance of classifying patients into different indication groups.

Except for one study by Urena et al. [9] and the high-risk population of Hutt et al. [10], the thromboembolic risk in the Austrian LAAC registry, as predicted by the $\mathrm{CHA}_{2} \mathrm{DS}_{2}$-VASc score (median 5) or $\mathrm{CHADS}_{2}$ score (median 3), was higher than in previous studies, which report a median $\mathrm{CHA}_{2} \mathrm{DS}_{2}$-VASc score of $4[3,11-17]$ or a median $\mathrm{CHADS}_{2}$ score of $2[18,19]$. The bleeding risk assessed by the HAS-BLED score was comparable to previous literature (median 3) [13-17].

To the authors' knowledge, this is the first study to stratify patients by the indication to perform LAAC. Previous studies only report scarce data. In four multicentre registries, $72-93 \%$ had a history of bleeding $[9,12,14,20]$. In the large EWOLUTION registry, only $31 \%$ of patients had a history of haemorrhage [3], and Kefer et al. only mentioned having included patients with a previous embolism despite being on an OAC, without giving distinct numbers [13]. Patient choice was the main indication for LAAC in $27.1 \%$ of patients in the Left-Atrium-Appendage Occluder Register-GErmany (LAARGE) [20]. In the two existing randomised controlled trials, all AF patients with an elevated stroke risk were evaluated for LAAC and only patients without a contraindication to an OAC were included [11,18].

\subsection{Procedural Outcome}

In Austria, both AmplatzerTM and Watchman ${ }^{\mathrm{TM}}$ devices were used for LAAC almost equally (52.2\% vs. $46.8 \%)$. Concomitant closure of the patient's foramen ovale was performed most frequently in the thromboembolism group, probably to avoid a further thromboembolism via a right-to-left shunt in this group with a history of recurrent thromboembolism. While Berti reported a much lower rate of concomitant procedures in the national Italian registry (2.4\%) [21], the rate of combined procedures in our analysis $(14.3 \%)$ was similar to other studies [22,23]. The procedural duration was similar to previous reports $[20,24,25]$.

With an incidence of 7.0\%, major procedural complications occurred fairly often in this high-risk patient population. It is noteworthy that there were also two ischemic strokes during the LAAC device implantation. We did not notice any differences in major or other complications between the indication groups. Cardiac tamponade was the most frequent major complication. Fortunately, all complications were managed successfully until discharge and the mortality until discharge was $0 \%$.

One reason for the comparatively high incidence of complications may have been the high-risk cohort undergoing LAAC. However, low operator experience may have also played a role, as the mean rate of procedures per year and centre was only 7.6. Interestingly, the centre with the lowest rate of yearly procedures (1.1) had the highest rate of periprocedural complications (25\%). It is to be discussed whether patients would benefit from a centralisation of LAAC to a few centres to optimise the procedural results and post-procedural follow-up.

The implantation success rate of $97.3 \%$ was comparable to other studies, which included either Watchman [3,11,12,18,19] or Amplatzer [9,13-17] devices, reporting a success rate of 90.9 [18] to $99.1 \%$ [17]. Major adverse events in the literature were similar to this analysis and ranged from 2.2 [15] to $12.0 \%[13]$.

\subsection{Post-Procedural Antithrombotic Treatment}

We found that post-procedural antithrombotic treatment was tailored in accordance with the patients' individual history. As a consequence, antithrombotic treatment after LAAC was very heterogeneous. For the first three months after the LAAC procedure, most of the patients received dual antiplatelet therapy. As a consequence of their medical history, the majority of patients in the thromboembolism group were on an OAC for 3-6 months after LAAC. However, despite their history of recurrent thromboembolism, even during OAC treatment, in almost 75\% of thromboembolism patients, no long-term OAC was prescribed, but $67 \%$ either received a single antiplatelet medication or no further antithrombotic medication. Interestingly, $12.4 \%$ of patients received no antithrombotic 
therapy at all at the time of discharge. This approach contradicts current guidelines [4], which endorse a course of single antiplatelet therapy for at least two weeks, even in patients with very high bleeding risk. However, in this analysis, there was no signal showing markedly increased thromboembolism rates in these patients.

In the entire population, long-term medical therapy mostly consisted of single antiplatelet therapy $(43.5 \%)$ or no therapy $(39.2 \%)$.

In the majority of the existing analyses, dual antiplatelet therapy was recommended for 1-6 months [9,12-14,16,17], followed by aspirin for 3 months [14,16] or indefinitely $[9,12,13]$. Warfarin was used for the first 45 days in Watchman Left Atrial Appendage System for Embolic Protection in Patients with Atrial Fibrillation (PROTECT AF) [18] and Watchman LAA Closure Device in Patients With Atrial Fibrillation Versus Long Term Warfarin Therapy (PREVAIL) studies [11], and in 27\% of patients in the EWOLUTION registry [3].

\subsection{Long-Term Outcome}

At the follow-up TOE, device-related complications, such as residual flow or thrombus formation, were rare (1.8\% and $1.9 \%$, respectively). Rehospitalisations due to the LAAC device itself were also rare $(1.7 \%)$, and there was only one case of a dislocation of the LAA occluder. However, follow-up TOE data were missing for a considerable number of patients $(40.3 \%)$, partly because the examination was performed in a department that was different from the implanting centre. This may have led to the under-reporting of long-term adverse events. Furthermore, regular follow-ups at the implanting centre may be beneficial after such a complicated procedure, as endorsed by guidelines [4].

During the first year of follow-up, $16.5 \%$ of patients experienced either death, stroke, peripheral thromboembolism, bleeding or an LAAC-associated hospitalisation. This fairly high long-term complication rate can be attributed to the high-risk patient population. When comparing the annual predicted thromboembolic and haemorrhagic events of historic controls to observed events in this study, a significant reduction of $57.0 \%$ of thromboembolic events was acknowledged, while the reduction of bleeding events $(30.2 \%)$ was not significant. The reduction of events was most pronounced in the thromboembolism group, with no embolic or bleeding events during the whole follow-up period. This fact is of interest, as all of these patients had a history of recurrent embolic events before LAAC and only a quarter received an OAC as an indefinite therapy after the procedure. However, the small sample size of this group limits the validity of these results. The group with the highest bleeding and thromboembolic event rates, despite a moderate baseline risk profile, was the other group with thromboembolic events in $4.5 \%$ and bleeding events in $5.7 \%$. This group also experienced the lowest reduction of events compared to historic controls. This fact might be explained by two reasons with unclear relevance: First, these patients could have more prevalent risk factors of bleeding or thromboembolism that are not addressed by traditional risk scores. Second, these patients might not profit as much from LAAC as other patients with a clear contraindication to an OAC. Further studies may be needed to further examine this heterogeneous subgroup. Due to the rather small sample size, only the reduction of annual embolic events of the whole population reached statistical significance $(p=0.035)$.

Because of the higher risk profile of our patient cohort, the thromboembolic rate of $3.7 \%$ was higher than in other studies, which reported events in 1.3 to $3.4 \%$ of patients $[9,11-16,18,26]$. The bleeding rate $(5.3 \%)$ was also higher than existing analyses or registries, which reported rates of $0.0-4.8 \%[9,13-16,18,26]$, possibly because of the excessively high prevalence of previous bleeding in this cohort. The reduction of bleeding and embolic events compared to historic controls has been confirmed in other studies [12,26], which reported a bleeding rate reduction of $46 \%$ [26] and a reduction of thromboembolic events of 77-83\% [12,26]. Concerning mortality, the results of this analysis (annual death rate $=8.6 \%$ ) were similar to other studies $(3.7$ to $10.8 \%)[9,11-16,18,26]$. 


\subsection{Limitations}

Large multi-centre real-world registries are valuable tools for monitoring the baseline factors and outcome of patients undergoing a procedure. We eliminated selection bias by including all patients undergoing LAAC in currently active centres in Austria. Due to external review at the majority of centres and the inclusion of data of the Austrian population registry, as well as searching the databases of hospital associations, we tried to minimise reporting bias and ensure a complete follow-up concerning mortality. However, the data quality cannot be compared to a controlled trial and a few patients had a follow-up of less than one year. In a proportion of patients, post-procedural TOE data was available because those patents were further managed at peripheral centres. Furthermore, the comparison with historic controls regarding the embolic and bleeding risk is always questionable, as risk scores never include all individual patient factors. This analysis only included Amplatzer ${ }^{\mathrm{TM}}$ and Watchman ${ }^{\mathrm{TM}}$ devices. The data may therefore not be extrapolated to other LAAC techniques, such as LAA suture ligation. Documentation of the procedural techniques was limited. Lastly, but importantly, the low sample size of the individual groups limited the validity of the results and may have led to an underestimation of factors that would have had a significant impact on outcome otherwise.

\section{Conclusions}

Patients undergoing LAAC in Austria have a high baseline risk of thromboembolic and bleeding events. While in a majority of patients, bleeding led to the decision to perform LAAC, other indications were heterogeneous, including thromboembolism, despite being on an OAC or patient preference. The LAAC procedure had a considerably high rate of short-term complications and a fairly elevated long-term complication rate. Long-term follow-ups suggested a reduction in thromboembolic events in the whole population when compared to historical controls.

Supplementary Materials: The following are available online at http://www.mdpi.com/2077-0383/9/10/3274/s1. Table S1: Preprocedural evaluation of treated patients, grouped by indication for LAAC. Table S2: Procedural outcome and follow-up echocardiography. Table S3: Post-procedural anticoagulation strategy. Table S4: Mean predicted annual stroke and bleeding events (as determined using $\mathrm{CHA}_{2} \mathrm{DS}_{2}$-VASc and HAS-BLED scores) compared to reported events in the Austrian LAAC Registry.

Author Contributions: Conceptualisation, D.Z., L.F. and D.S.; data curation, D.Z.; formal analysis, D.Z.; funding acquisition, D.S.; investigation, D.Z., R.S., L.F., G.G.T., O.L., G.S., H.G., P.V., B.L., A.S., G.D.-K., M.P., J.A., W.T., C.S., K.S., R.K.B., F.B., S.M., N.V., K.A., A.Z. and D.S.; methodology, D.Z., R.S., M.R. and D.S.; project administration, D.S.; resources, D.Z. and D.S.; software, D.Z.; supervision, D.S.; validation, D.Z., L.F., G.G.T., O.L., H.G., P.V., B.L., A.S., G.D.-K., M.P., J.A., W.T., C.S., K.S., R.K.B., M.R., F.B., S.M., N.V., K.A., A.Z. and D.S.; visualisation, D.Z. and G.S.; writing—original draft, D.Z.; writing—review and editing, D.Z., R.S., L.F., G.G.T., O.L., G.S., H.G., P.V., B.L., A.S., G.D.-K., M.P., J.A., W.T., C.S., K.S., R.K.B., M.R., F.B., S.M., N.V., K.A., A.Z. and D.S. All authors have read and agreed to the published version of the manuscript.

Funding: This research was supported by an unrestricted grant from Boston Scientific TM (Malborough, MA, USA).

Acknowledgments: We owe our sincere gratitude to all colleagues of the participating centres for their collaboration.

Conflicts of Interest: H.G. serves as a consultant for Abbott. G.S. received speaker honoraria from Bayer, Biotronik and Boston Scientific. R.K.B. serves as proctor for Boston Scientific and has received grants from Abbott. D.S. received speaker honoraria from Boston Scientific. All other authors have nothing to disclose with respect to this publication.

\section{References}

1. Kirchhof, P.; Benussi, S.; Kotecha, D.; Ahlsson, A.; Atar, D.; Casadei, B.; Castella, M.; Diener, H.C.; Heidbuchel, H.; Hendriks, J.; et al. 2016 ESC Guidelines for the management of atrial fibrillation developed in collaboration with EACTS. Europace 2016, 18, 1609-1678. [CrossRef]

2. Meschia, J.F.; Bushnell, C.; Boden-Albala, B.; Braun, L.T.; Bravata, D.M.; Chaturvedi, S.; Creager, M.A.; Eckel, R.H.; Elkind, M.S.; Fornage, M.; et al. Guidelines for the primary prevention of stroke: A statement for healthcare professionals from the American Heart Association/American Stroke Association. Stroke 2014, 45, 3754-3832. [CrossRef] 
3. Boersma, L.V.; Schmidt, B.; Betts, T.R.; Sievert, H.; Tamburino, C.; Teiger, E.; Pokushalov, E.; Kische, S.; Schmitz, T.; Stein, K.M.; et al. Implant success and safety of left atrial appendage closure with the WATCHMAN device: Peri-procedural outcomes from the EWOLUTION registry. Eur. Heart J. 2016, 37, 2465-2474. [CrossRef]

4. Glikson, M.; Wolff, R.; Hindricks, G.; Mandrola, J.; Camm, A.J.; Lip, G.Y.H.; Fauchier, L.; Betts, T.R.; Lewalter, T.; Saw, J.; et al. EHRA/EAPCI expert consensus statement on catheter-based left atrial appendage occlusion-An update. Europace 2020, 22, 184. [CrossRef]

5. Lebhart, G.; Neustädter, C.; Kytir, J. The new Population Register at Statistics Austria: Conceptualization and Methodology for Register-based Flow and Stock Statistics. Austrian J. Stat. 2007, 36, 277-289. [CrossRef]

6. Olesen, J.B.; Lip, G.Y.; Hansen, M.L.; Hansen, P.R.; Tolstrup, J.S.; Lindhardsen, J.; Selmer, C.; Ahlehoff, O.; Olsen, A.M.; Gislason, G.H.; et al. Validation of risk stratification schemes for predicting stroke and thromboembolism in patients with atrial fibrillation: Nationwide cohort study. BMJ 2011, 342, d124. [CrossRef]

7. Olesen, J.B.; Lip, G.Y.; Hansen, P.R.; Lindhardsen, J.; Ahlehoff, O.; Andersson, C.; Weeke, P.; Hansen, M.L.; Gislason, G.H.; Torp-Pedersen, C. Bleeding risk in 'real world' patients with atrial fibrillation: Comparison of two established bleeding prediction schemes in a nationwide cohort. J. Thromb. Haemost. 2011, 9, 1460-1467. [CrossRef]

8. LaHaye, S.A.; Gibbens, S.L.; Ball, D.G.; Day, A.G.; Olesen, J.B.; Skanes, A.C. A clinical decision aid for the selection of antithrombotic therapy for the prevention of stroke due to atrial fibrillation. Eur. Heart J. 2012, 33, 2163-2171. [CrossRef]

9. Urena, M.; Rodes-Cabau, J.; Freixa, X.; Saw, J.; Webb, J.G.; Freeman, M.; Horlick, E.; Osten, M.; Chan, A.; Marquis, J.F.; et al. Percutaneous left atrial appendage closure with the AMPLATZER cardiac plug device in patients with nonvalvular atrial fibrillation and contraindications to anticoagulation therapy. J. Am. Coll. Cardiol. 2013, 62, 96-102. [CrossRef]

10. Hutt, E.; Wazni, O.M.; Kaur, S.; Saliba, W.I.; Tarakji, K.G.; Kapadia, S.; Aguilera, J.; Barakat, A.F.; Abdallah, M.; Jaber, W.; et al. Left Atrial Appendage Closure Device Implantation in Patients at Very High Risk for Stroke. Heart Rhythm 2019. [CrossRef]

11. Holmes, D.R., Jr.; Kar, S.; Price, M.J.; Whisenant, B.; Sievert, H.; Doshi, S.K.; Huber, K.; Reddy, V.Y. Prospective randomized evaluation of the Watchman Left Atrial Appendage Closure device in patients with atrial fibrillation versus long-term warfarin therapy: The PREVAIL trial. J. Am. Coll. Cardiol. 2014, 64, 1-12. [CrossRef]

12. Reddy, V.Y.; Mobius-Winkler, S.; Miller, M.A.; Neuzil, P.; Schuler, G.; Wiebe, J.; Sick, P.; Sievert, H. Left atrial appendage closure with the Watchman device in patients with a contraindication for oral anticoagulation: The ASAP study (ASA Plavix Feasibility Study With Watchman Left Atrial Appendage Closure Technology). J. Am. Coll. Cardiol. 2013, 61, 2551-2556. [CrossRef]

13. Kefer, J.; Vermeersch, P.; Budts, W.; Depotter, T.; Aminian, A.; Benit, E.; Stammen, F. Transcatheter left atrial appendage closure for stroke prevention in atrial fibrillation with Amplatzer cardiac plug: The Belgian Registry. Acta Cardiol. 2013, 68, 551-558. [CrossRef]

14. Lopez-Minguez, J.R.; Nogales-Asensio, J.M.; Infante De Oliveira, E.; De Gama Ribeiro, V.; Ruiz-Salmeron, R.; Arzamendi-Aizpurua, D.; Costa, M.; Gutierrez-Garcia, H.; Fernandez-Diaz, J.A.; Martin-Yuste, V.; et al. Long-term Event Reduction After Left Atrial Appendage Closure. Results of the Iberian Registry II. Rev. Esp. Cardiol. 2019, 72, 449-455. [CrossRef]

15. Santoro, G.; Meucci, F.; Stolcova, M.; Rezzaghi, M.; Mori, F.; Palmieri, C.; Paradossi, U.; Pastormerlo, L.E.; Rosso, G.; Berti, S. Percutaneous left atrial appendage occlusion in patients with non-valvular atrial fibrillation: Implantation and up to four years follow-up of the AMPLATZER Cardiac Plug. EuroIntervention 2016, 11, 1188-1194. [CrossRef]

16. Tzikas, A.; Shakir, S.; Gafoor, S.; Omran, H.; Berti, S.; Santoro, G.; Kefer, J.; Landmesser, U.; Nielsen-Kudsk, J.E.; Cruz-Gonzalez, I.; et al. Left atrial appendage occlusion for stroke prevention in atrial fibrillation: Multicentre experience with the AMPLATZER Cardiac Plug. EuroIntervention 2016, 11, 1170-1179. [CrossRef]

17. Landmesser, U.; Tondo, C.; Camm, J.; Diener, H.C.; Paul, V.; Schmidt, B.; Settergren, M.; Teiger, E.; Nielsen-Kudsk, J.E.; Hildick-Smith, D. Left atrial appendage occlusion with the AMPLATZER Amulet device: One-year follow-up from the prospective global Amulet observational registry. EuroIntervention 2018, 14, e590-e597. [CrossRef] 
18. Holmes, D.R.; Reddy, V.Y.; Turi, Z.G.; Doshi, S.K.; Sievert, H.; Buchbinder, M.; Mullin, C.M.; Sick, P.; Investigators, P.A. Percutaneous closure of the left atrial appendage versus warfarin therapy for prevention of stroke in patients with atrial fibrillation: A randomised non-inferiority trial. Lancet 2009, 374, 534-542. [CrossRef]

19. Reddy, V.Y.; Holmes, D.; Doshi, S.K.; Neuzil, P.; Kar, S. Safety of percutaneous left atrial appendage closure: Results from the Watchman Left Atrial Appendage System for Embolic Protection in Patients with AF (PROTECT AF) clinical trial and the Continued Access Registry. Circulation 2011, 123, 417-424. [CrossRef]

20. Fastner, C.; Nienaber, C.A.; Park, J.W.; Brachmann, J.; Zeymer, U.; Goedde, M.; Sievert, H.; Geist, V.; Lewalter, T.; Krapivsky, A.; et al. Impact of left atrial appendage morphology on indication and procedural outcome after interventional occlusion: Results from the prospective multicentre German LAARGE registry. EuroIntervention 2018, 14, 151-157. [CrossRef]

21. Berti, S.; Pastormerlo, L.E.; Santoro, G.; Brscic, E.; Montorfano, M.; Vignali, L.; Danna, P.; Tondo, C.; Rezzaghi, M.; D'Amico, G.; et al. Intracardiac Versus Transesophageal Echocardiographic Guidance for Left Atrial Appendage Occlusion: The LAAO Italian Multicenter Registry. JACC Cardiovasc. Interv. 2018, 11, 1086-1092. [CrossRef] [PubMed]

22. Saw, J.; Tzikas, A.; Shakir, S.; Gafoor, S.; Omran, H.; Nielsen-Kudsk, J.E.; Kefer, J.; Aminian, A.; Berti, S.; Santoro, G.; et al. Incidence and Clinical Impact of Device-Associated Thrombus and Peri-Device Leak Following Left Atrial Appendage Closure With the Amplatzer Cardiac Plug. JACC Cardiovasc. Interv. 2017, 10, 391-399. [CrossRef] [PubMed]

23. Wolfrum, M.; Attinger-Toller, A.; Shakir, S.; Gloekler, S.; Seifert, B.; Moschovitis, A.; Khattab, A.; Maisano, F.; Meier, B.; Nietlispach, F. Percutaneous left atrial appendage occlusion: Effect of device positioning on outcome. Catheter. Cardiovasc. Interv. 2016, 88, 656-664. [CrossRef] [PubMed]

24. Berti, S.; Santoro, G.; Brscic, E.; Montorfano, M.; Vignali, L.; Danna, P.; Tondo, C.; D’Amico, G.; Stabile, A.; Sacca, S.; et al. Left atrial appendage closure using AMPLATZER devices: A large, multicenter, Italian registry. Int. J. Cardiol. 2017, 248, 103-107. [CrossRef] [PubMed]

25. Teiger, E.; Thambo, J.B.; Defaye, P.; Hermida, J.S.; Abbey, S.; Klug, D.; Juliard, J.M.; Pasquie, J.L.; Rioufol, G.; Lepillier, A.; et al. Percutaneous Left Atrial Appendage Closure Is a Reasonable Option for Patients With Atrial Fibrillation at High Risk for Cerebrovascular Events. Circ. Cardiovasc. Interv. 2018, 11, e005841. [CrossRef] [PubMed]

26. Boersma, L.V.; Ince, H.; Kische, S.; Pokushalov, E.; Schmitz, T.; Schmidt, B.; Gori, T.; Meincke, F.; Protopopov, A.V.; Betts, T.; et al. Evaluating Real-World Clinical Outcomes in Atrial Fibrillation Patients Receiving the WATCHMAN Left Atrial Appendage Closure Technology. Circ. Arrhythm. Electrophysiol. 2019, 12, e006841. [CrossRef] [PubMed]

(C) 2020 by the authors. Licensee MDPI, Basel, Switzerland. This article is an open access article distributed under the terms and conditions of the Creative Commons Attribution (CC BY) license (http://creativecommons.org/licenses/by/4.0/). 